\title{
Seeking key microorganisms for enhancing methane production in anaerobic digestion of waste sewage sludge
}

\begin{abstract}
Efficient approaches for the utilization of waste sewage sludge have been widely studied. One of them is to use it for the bioenergy production, specifically methane gas which is well-known to be driven by complex bacterial interactions during the anaerobic digestion process. Therefore, it is important to understand not only microorganisms for producing methane but also those for controlling or regulating the process. In this study, azithromycin analogs belonging to macrolide, ketolide, and lincosamide groups were applied to investigate the mechanisms and dynamics of bacterial community in waste sewage sludge for methane production. The stages of anaerobic digestion process were evaluated by measuring the production of intermediate substrates, such as protease activity, organic acids, the quantification of bacteria and archaea, and its community dynamics. All azithromycin analogs used in this study achieved a high methane production compared to the control sample without any antibiotic due to the efficient hydrolysis process and the presence of important fermentative bacteria and archaea responsible in the methanogenesis stage. The key microorganisms contributing to the methane production may be Clostridia, Cladilinea, Planctomycetes, and Alphaproteobacteria as an accelerator whereas Nitrosomonadaceae and Nitrospiraceae may be suppressors for methane production. In conclusion, the utilization of antibiotic analogs of macrolide, ketolide, and lincosamide groups has a promising ability in finding the essential microorganisms and improving the methane production using waste sewage sludge.
\end{abstract}

Keyword: Ketolide; Lincosamide; Macrolide; Methane; Microbial community; Sewage sludge 